No. 4100 May 29, 1948

\section{Total Emission Damping}

IN recent letters in Nature, C. N. Smyth ${ }^{1}$, A. van der $\mathrm{Ziel}^{2}$ and A. van der Ziel and A. Versnel ${ }^{3}$ have reported measurements on the damping produced by a diode in the non-conducting state placed across a resonant circuit.

To investigate this subject mathematically, the following idealization has been employed. It is assumed that the thermionic electrons are all emitted with speed $v_{0}$ at right angles to the cathode surface, and that they are then acted upon by a constant retarding field $E_{R}$ superimposed upon the small alternating field $E_{S} \sin (\omega t+\varphi)$. The time of flight of these electrons in the absence of the alternating field is then $2 v_{0} / E_{R} \frac{e}{m}=T$, and the transit angle is $\theta=\omega T$. If $E_{S}$ is small compared with $E_{R}$, it can then be shown that the damping conductance $1 / R$ is given by

$$
\frac{1}{R}=\frac{8 V_{0} I}{V_{R^{2}}} f(\theta)
$$

In this equation $V_{0} I$ is total power emitted by the cathode, that is, the product of the number of electrons emitted per second and the kinetic energy of each electron. $V_{R}$ is $E_{R} D$, where $D$ is the effective distance across which the constant fields $E_{R}$ and $E_{S}$ are assumed to act.

The interesting part of equation $(1)$ is $f(\theta)$, which is

$$
\begin{aligned}
& f(\theta)=\frac{1}{\theta^{2}}\left[\left(1-\frac{1}{\theta^{2}}\right)\right. \\
& \left\{4(1-\cos \theta)-4 \theta \sin \theta+\theta^{2}(1+\cos \theta)\right\}+ \\
& \{2(1-\cos \theta)-\theta \sin \theta)\}] \text {. }
\end{aligned}
$$

This may be compared with the corresponding function for the conducting diode, $F(\theta)$, where

$$
F(\theta)=\frac{1}{\theta^{2}}[2(1-\cos \theta)-\theta \sin \theta] \text {. }
$$

Graphs of $f(\theta)$ and $F(\theta)$ are shown in Fig. 1.

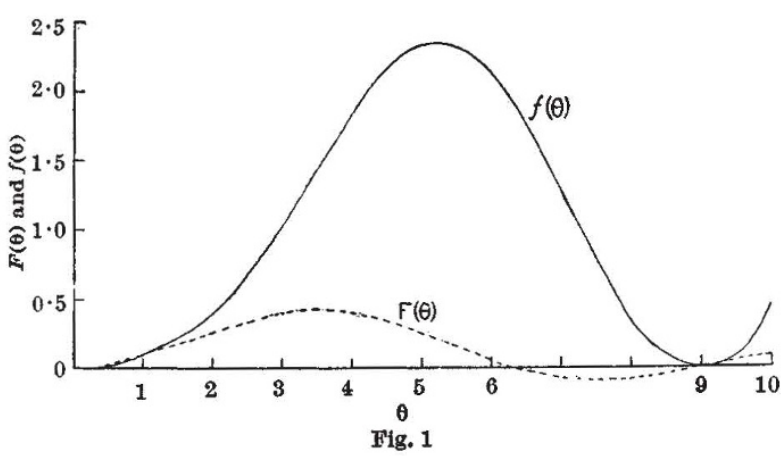

That the absolute magnitude of the damping in equation (1) can be of the correct order of magnitude may be shown as follows. In Smyth's case ${ }^{1}$, assume the values $V_{R}=1, V_{0}=1 / 16$, both in volts, and $I=1 / 10$ amp. per cm. ${ }^{2}$. Then equation (1) gives $R=20 \mathrm{ohms}$ per $\mathrm{cm} .^{2}$. In van der Ziel's case ${ }^{2}$, the damping is so small and the cathode-anode spacing is so large that it would be unwise to guess the value of $V_{R}$.

An interesting point emerges if equation (l) is written in the correct form to exhibit the variation of the damping with $V_{\boldsymbol{R}}$, where $V_{R}$ is assumed to be proportional to $1 / \theta$. Then

$$
\frac{1}{R}=\frac{I}{(\omega D)^{2}} \theta^{2} f(\theta)=\frac{I}{(\omega D)^{2}} \frac{\theta^{4}}{9}
$$

for small values of $\theta$. The function $\theta^{2} f(\theta)$ is shown in Fig. 2.

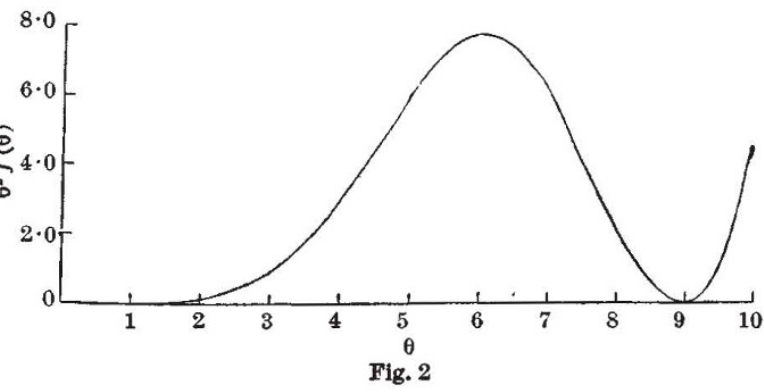

It is not suggested that the idealization which has led to the results reported above is adequate to explain all the phenomena of total emission damping; but it points to a mode of attack on the problem which is hopeful. The method by which equations (1) and (2) are derived will be published elsewhere in due course.

Admiralty Signal and Radar Establishment,

\section{J. Thomson}

Haslemere, Surrey. Feb. 2.

${ }^{1}$ Smyth, C. N., Nature, 157, 841 (1946).

' van der Ziel, A., Nature, 159, 677 (1947).

'van der Ziel, A., and Versnel, A., Nature, 159, 640 (1947).

\section{Theory of the Streaming Potential}

BоoTH $^{1}$ has recently pointed out the unsatisfactory nature of the existing theory of electrokinetics, and suggested modifications to the commonly used equations of Helmholtz ${ }^{2}$ and Smoluchowski ${ }^{3}$, with particular reference to the phenomenon of electrophoresis. I have devoted some attention to the theory of the streaming potential difference set up in the plane of flow when an electrical double layer, existing at an immobile interface in an ionic liquid, is sheared. This potential difference will tend to resist the flow of the liquid owing to the electrical retarding force acting on the ions of the double layer. It has been shown ${ }^{4,5}$ that in narrow channels this electrical resistance to flow, or 'electroviscous resistance', can assume considerable importance, with the result that the apparent viscosity of a liquid in such channels may be increased several fold, depending on the values of the electrokinetic potential of the double layer, the specific conductivity of the liquid in the channel, and the dielectric constant. Various workers ${ }^{6-8}$ have reported the qualitative observation of such an effect, and a quantitative experimental examination of the subject has been commenced in this laboratory.

In view of this apparent increase in viscosity in narrow channels, it is to be expected that zetapotentials calculated from measurements made in diaphragms of very small particle size will lead to anomalously low results if the usual Helmholtz Smoluchowski equation is used. The results of Bull and Gortner ${ }^{9}$ and others ${ }^{10-12}$ have been explained on this basis ${ }^{4}, 5$.

The results of this analysis are of importance when applied to the theories of surface conductivity and of lyophobic colloid stability, and offer an explanation of certain anomalous results obtained by workers ${ }^{13-15}$ 